\title{
Feeding Mathematical Methods into Engineering Courses
}

\author{
Zhidong $\mathrm{Hu}$ \\ College of Engineering and Technology, Northeast Forestry University Harbin, Heilongjiang, China
}

\author{
Keywords: Mathematical methods; ODE; PDE; Matrix
}

\begin{abstract}
The students in engineering coursed are short of mathematics knowledge to solve engineering problems. In order to strengthen the mathematical knowledge of students in engineering courses, the mathematical methods are provided in the paper. Based on the author's research work, the explicit Euler's method, implicit Euler's method, modified Euler's method; runge - kutta method and ODE45 method are introduced and compared for solving the ODE problem. All these methods are consistent with each other in solving the $2^{\text {nd }}$ order ODE. The error of them is very small. Also PDE method is introduced for solving PDE equations. And finite element method (FEM) is implied in modeling the problem. These methods are useful for students to build up the model to describe and solve the technical problems. This can also provide guidance for the future learning and teaching of engineering courses.
\end{abstract}

\section{Introduction}

In order to describe clearly about the mathematical methods used in engineering courses, an example based on author's research is provided in the paper. In the past century, cavitation has been identified as one of the most important causes of hydraulic machinery erosion, and a great amount of experimental and numerical research has been done in order to investigate the erosion mechanism caused by cavitation. The earliest work was done by Rayleigh [1] for a spherical vapor bubble, in which it was thought that the high pressure around a collapsing bubble was the reason for the erosion caused by cavitation. Equation (1) is the classic bubble motion equation deduced by Rayleigh.

$$
r \frac{3}{2} \&=\frac{p_{B}-p_{\infty}}{\rho}
$$

Where:

$\begin{array}{lll}r & - & \text { radius of the bubble }(\mathrm{m}) ; \\ P_{\mathrm{B}} & - & \text { pressure within the bubble }(\mathrm{Pa}) ; \\ p_{\infty} & - & \begin{array}{l}\text { external pressure infinitely far from the bubble }(\mathrm{Pa}) \\ \rho\end{array} \\ \begin{array}{l}\text { density of the surrounding liquid }\left(\mathrm{kg} / \mathrm{m}^{3}\right) ; \\ t\end{array} & - & \text { time }(\mathrm{s}) .\end{array}$

\section{Dimensional Analysis Method}

The International System of Units (SI) defines seven units of measure as a basic set from which all other SI units are derived. The SI base units and their physical quantities are: meter $(\mathrm{m})$ for length, kilogram (kg) for mass, second (s) for time, ampere (A) for electric current, Kelvin (K) temperature, candela (cd) for luminous intensity and mole (mol) for amount of substance. Length, kilogram and time are three commonly used units in engineering courses.

In engineering and science, dimensional analysis is the analysis of the relationships between different physical quantities by identifying their fundamental dimensions and units of measure and tracking these dimensions as calculations or comparisons are performed. Converting from one dimensional unit to another is often somewhat complex. Dimensional analysis, or more specifically the factor-label method, also known as the unit-factor method, is a widely used technique for such conversions using the rules of algebra. 
So the dimensional analysis expression of equation (1) can be written as:

$$
m \frac{m}{s^{2}}+\frac{3}{2}\left(\frac{m}{s}\right)^{2}=\frac{\frac{k g \frac{m}{s^{2}}}{\frac{m^{2}}{k g}}}{\frac{m^{3}}{s}} \propto\left(\frac{m}{s}\right)^{2}
$$

Using this method, the correctness of derivation can be easily guaranteed. And the characteristics and essence of the equation is very clearly to read.

\section{Ode Solution Methods}

In mathematics, an ordinary differential equation (ODE) is a differential equation containing one or more functions of one independent variable and its derivatives. Ordinary differential equations (ODEs) arise in many contexts of mathematics, engineering and science. Mathematical descriptions of change use differentials and derivatives. Various differentials, derivatives, and functions become related to each other via equations, and thus a differential equation is a result that describes dynamically changing phenomena, evolution, and variation. Often, quantities are defined as the rate of change of other quantities, or gradients of quantities, which is how they enter differential equations.

If the pressure difference in equation (1) is chosen as fixed value, it belongs to ODE problem. The explicit Euler's method, implicit Euler's method, Modified Euler's method, ODE45 method and Runge-Kutta method are used for solving this ODE problem [2].

The expression of explicit Euler's method is:

$$
r_{i+1}=r_{i}+h \times f\left(t_{i}, r_{i}\right)
$$

The expression of implicit Euler's method is:

$$
r_{i+1}=r_{i}+h \times f\left(t_{i+1}, r_{i+1}\right)
$$

The expression of modified Euler's method is:

$$
r_{i+1}=r_{i}+\frac{h}{2} \times\left[f\left(t_{i+1}, r_{i+1}\right)+f\left(t_{i}, r_{i}\right)\right]
$$

The expression of 4-stage Runge-Kutta method is:

$$
r_{i+1}=r_{i}+\frac{h}{6} \times\left[K_{1}+2 K_{2}+2 K_{3}+K_{4}\right]
$$

And the parameters are calculated as:

$$
\left\{\begin{array}{l}
K_{1}=f\left(t_{i}, r_{i}\right) \\
K_{2}=f\left(t_{i}+\frac{h}{2}, r_{i}+\frac{h}{2} K_{1}\right) \\
K_{3}=f\left(t_{i}+\frac{h}{2}, r_{i}+\frac{h}{2} K_{2}\right) \\
K_{4}=f\left(t_{i}+h, r_{i}+h K_{3}\right)
\end{array}\right.
$$

Where: $h$ - step size.

The ODE45 method is an embedded order in MATLAB program.

First the dynamic growing stage of single bubble is analyzed using above methods. 


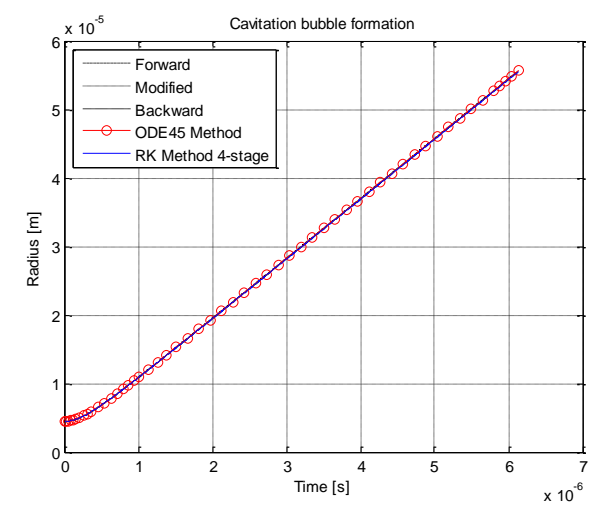

Bubble radius during growing stage

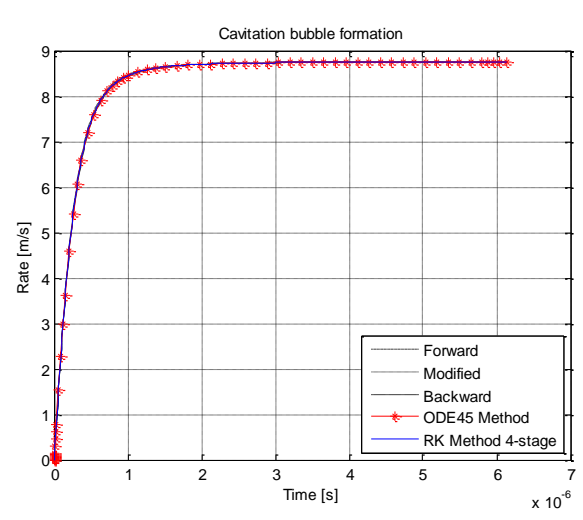

Growing rate

Figure 1. Dynamic growing analysis of bubble

Secondly, the dynamic collapse stage of single bubble is analyzed using above methods.

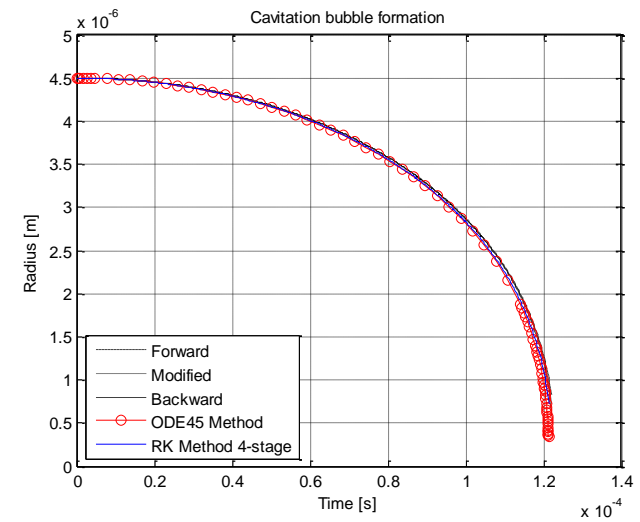

Bubble radius during collapse stage

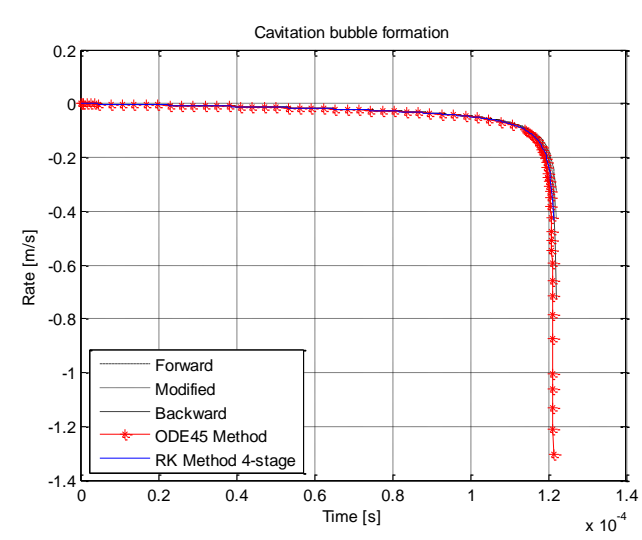

Collapse rate

Figure 2. Dynamic collapse analysis of bubble

The conclusion is that, all these five methods are very consistent with each other in analyzing the single bubble dynamics. The error between them is very small.

\section{Matrix Method}

Matrix theory is an important branch of mathematics and the main content of linear algebra. A matrix is defined as a rectangular array of numbers, symbols, or expressions, arranged in rows and columns. But the matrix is very abstract for understanding. In order to have a good comprehension of matrix, another example is provided below.

In order to analyze the pressure distribution when the two parallel plates filled with oil with initial gap separated from each other, the model is built up as following.

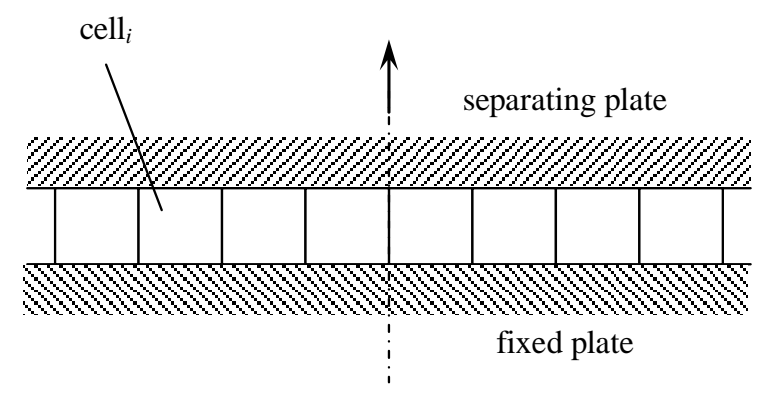

Figure 3. Separation model of two parallel plates filled with oil 
The pressure distribution is analyzed during the separation process. First the theoretical method and matrix are used for this analysis. For the $i^{\text {th }}$ cell, the dynamic equation can be expressed as:

$$
\frac{p_{i+1}-2 p_{i}+p_{i-1}}{\Delta x^{2}} \times \frac{h^{3}(t)}{12 \eta}=\frac{\partial h(t)}{\partial t}
$$

Where: $h(t)$ - separation height function of time $t$;

$\eta$ - kinematic viscosity of the surrounding liquid $(\mathrm{Pa} \cdot \mathrm{s})$.

$$
\left[\begin{array}{ccccccc}
1 & 0 & 0 & & \mathrm{~L} & & 0 \\
2 & -3 & 1 & & & \mathrm{O} & \\
0 & 1 & -2 & 1 & \mathrm{O} & & \mathrm{M} \\
& 0 & 1 & -2 & 1 & & \\
\mathrm{M} & & \mathrm{O} & \mathrm{O} & \mathrm{O} & \mathrm{O} & 0 \\
& & \mathrm{~L} & 0 & 1 & -2 & 1 \\
0 & & \mathrm{~L} & & 0 & 1 & -1
\end{array}\right]\left[\begin{array}{l}
p_{0} \\
p_{1} \\
p_{2} \\
p_{3} \\
\mathrm{M} \\
p_{N-1} \\
p_{N}
\end{array}\right]=a_{1}\left[\begin{array}{l}
p_{0} / a_{1} \\
1 \\
1 \\
1 \\
\mathrm{M} \\
1 \\
1
\end{array}\right]
$$

It can be simplified as:

$$
\stackrel{\vee}{A} \stackrel{\vee}{P}=a_{1} \stackrel{v}{B}
$$

Where: $a_{1}=\frac{12 \Delta x^{2} \eta \boldsymbol{k}(t)}{h^{3}(t)}, \underset{A}{\mathrm{v}}=\left[\begin{array}{ccccccc}1 & 0 & 0 & & \mathrm{~L} & & 0 \\ 2 & -3 & 1 & & & \mathrm{O} & \\ 0 & 1 & -2 & 1 & 0 & & \mathrm{M} \\ & 0 & 1 & -2 & 1 & & \\ \mathrm{M} & \mathrm{O} & 0 & 0 & 0 & 0 \\ & \mathrm{~L} & 0 & 1 & -2 & 1 \\ 0 & & \mathrm{~L} & & 0 & 1 & -1\end{array}\right] \stackrel{\mathrm{v}}{P}=\left[\begin{array}{l}p_{0} \\ p_{1} \\ p_{2} \\ p_{3} \\ \mathrm{M} \\ p_{N-1} \\ p_{N}\end{array}\right], \underset{B}{\mathrm{v}}=\left[\begin{array}{l}p_{0} / a_{1} \\ 1 \\ 1 \\ 1 \\ \mathrm{M} \\ 1 \\ 1\end{array}\right]$

So through the matrix the relationship between pressure distribution and position can be described well. As the time changing, the pressure changes with time and position.
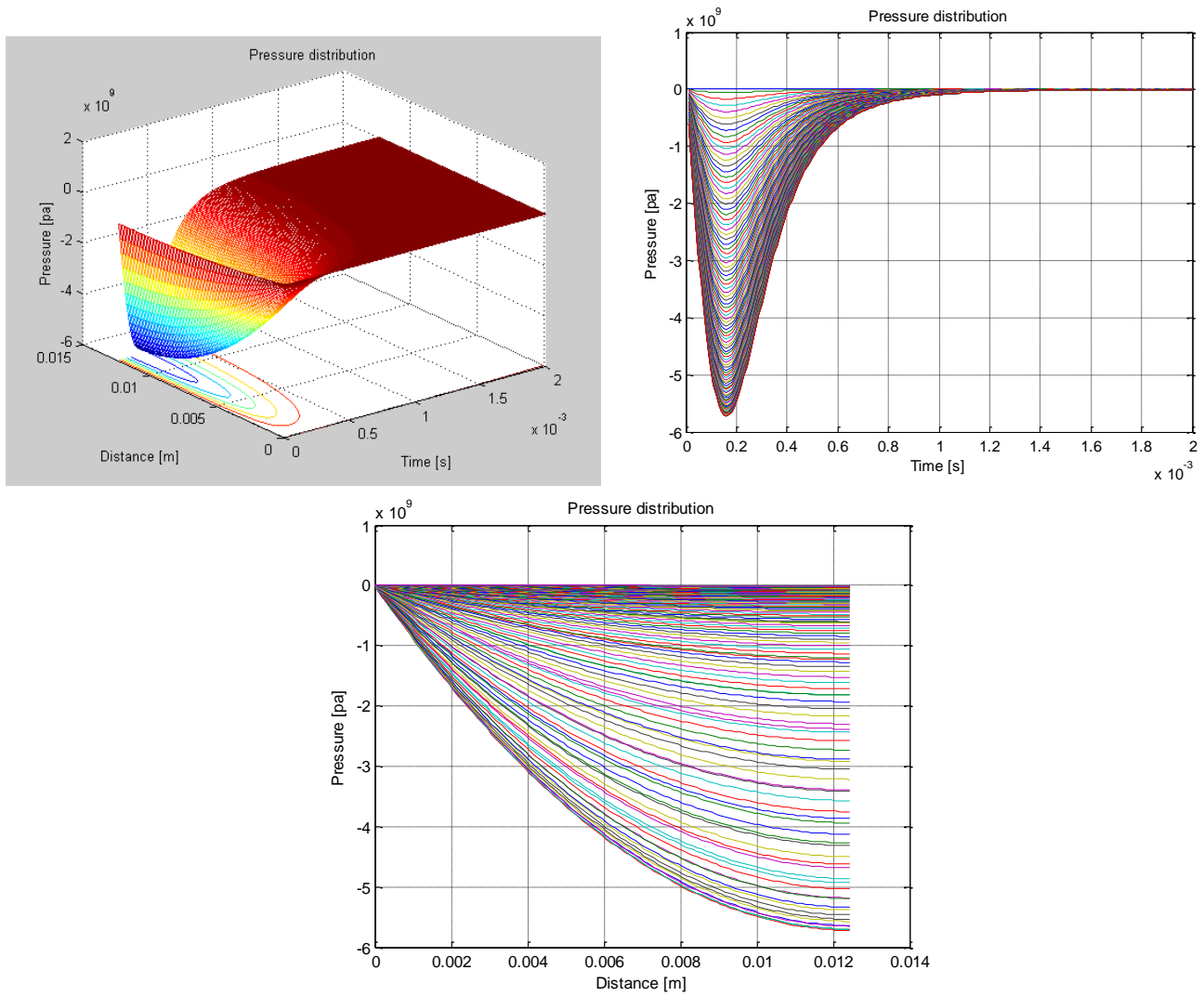

Figure 4. Pressure distribution using theoretical method 


\section{Pde Solution Methods}

In mathematics, a partial differential equation (PDE) is a differential equation that contains unknown multivariable functions and their partial derivatives. PDEs are used to formulate problems involving functions of several variables, and are either solved by hand, or used to create a relevant computer model.

Also, aiming at the separation model of tow parallel plates filled with oil, the Matlab software provides a PDE tool 'pdepe' for solving the PDE equations. The initial condition (IC) and boundary condition (BC) are required for PDE solution [3-5].

$$
\begin{aligned}
& C\left(x, t, u, \frac{\partial u}{\partial x}\right) \frac{\partial u}{\partial t}= \\
& x^{-m} \frac{\partial}{\partial x}\left(x^{m} f\left(x, t, u, \frac{\partial u}{\partial x}\right)\right)+S\left(x, t, u, \frac{\partial u}{\partial x}\right)
\end{aligned}
$$

Let $\mathrm{m}=0, x \in[0,0.0125], t \in[0,0.002], f\left(x, t, u, \frac{\partial u}{\partial x}\right)=\frac{\partial p}{\partial x}, C\left(x, t, u, \frac{\partial u}{\partial x}\right)=0$,

$S\left(x, t, u, \frac{\partial u}{\partial x}\right)=-\frac{\partial h(t)}{\partial t} \frac{12 \eta}{h^{3}(t)}, I C: p(x, 0)=1$ bar $, B C:\left\{\begin{array}{l}p(0, t)=1 \text { bar } \\ p(0.0125, t)=1 b a r\end{array}\right.$
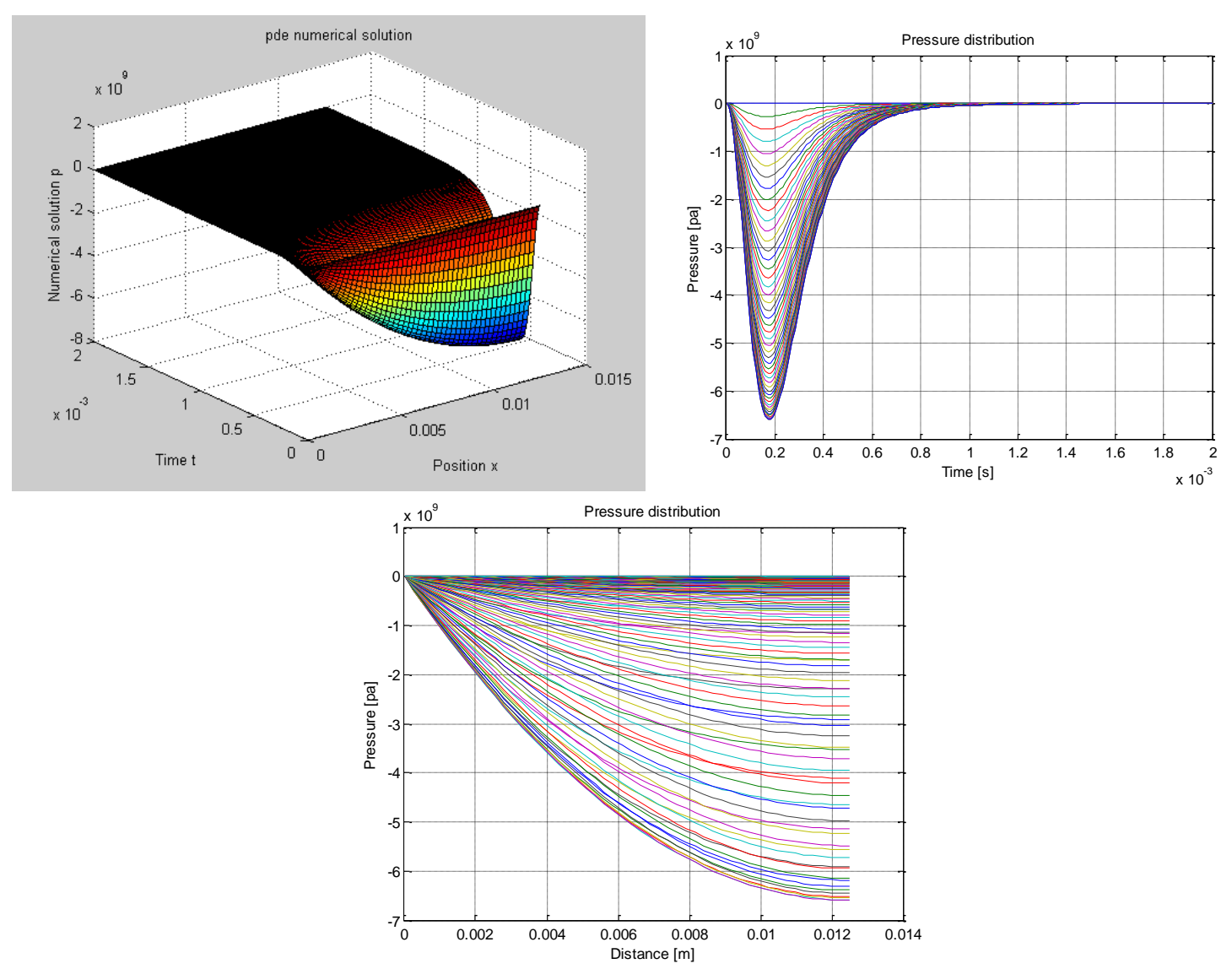

Figure 5. Pressure distribution with pdepd method

So the results are consistent with the theoretical analysis.

Combining the cavitation bubble dynamics and pressure distribution together, the model can be built like Fig. 6. 


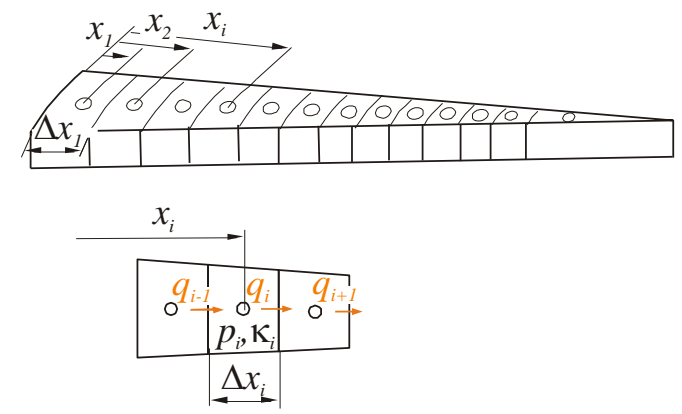

Figure 6. Separation model with cavitation bubbles

And viscosity of oil is also taken into account, so the equations are written as (12) and (13).

$r \frac{3}{2} \&+4 \frac{\eta \&}{\rho r}=\frac{p_{B}-p_{\infty}}{\rho}$

$$
\left[\begin{array}{ccccccc}
1 & 0 & 0 & & \mathrm{~L} & & 0 \\
2 & -3 & 1 & & & \mathrm{O} & \\
0 & 1 & -2 & 1 & \mathrm{O} & & \mathrm{M} \\
& 0 & 1 & -2 & 1 & & \\
\mathrm{M} & \mathrm{O} & \mathrm{O} & \mathrm{O} & \mathrm{O} & 0 \\
& & \mathrm{~L} & 0 & 1 & -2 & 1 \\
0 & \mathrm{~L} & & 0 & 1 & -1
\end{array}\right]\left[\begin{array}{l}
p_{0} \\
p_{1} \\
p_{2} \\
p_{3} \\
\mathrm{M} \\
p_{N-1} \\
p_{N}
\end{array}\right]=a_{1}\left[\begin{array}{l}
p_{0} / a_{1} \\
1 \\
1 \\
1 \\
\mathrm{M} \\
1 \\
1
\end{array}\right]-a_{2}\left[\begin{array}{l}
0 \\
r_{1}^{2} \\
r_{2}^{2} \\
r_{3}^{2} \\
\mathrm{M} \\
r_{N-1}^{2} \\
r_{N}^{2}
\end{array}\right]
$$

The simplified expression is:

$$
\stackrel{\vee}{A} \stackrel{\vee}{P}=a_{1} \stackrel{\vee}{B}-a_{2} \stackrel{\vee}{C}
$$

Where: $a_{2}=\frac{48 \pi \Delta x \eta}{b h^{3}(t)}, \underset{\mathrm{v}}{\mathrm{v}}=\left[\begin{array}{l}0 \\ r_{1}^{2} \\ r_{2}^{2} \\ r_{3}^{2} \\ \mathrm{M} \\ r_{N-1}^{2} \\ r_{N}^{2}\end{array}\right]$

So the complete life of each bubble in each cell can be obtained.

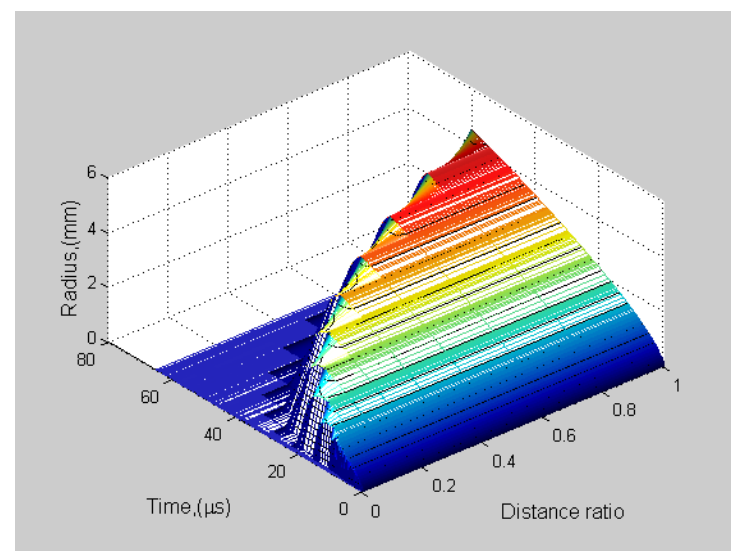

Figure 7. Dynamic bubble life during separation process 


\section{Conclusions}

During the separation process of two plates filled with oil, the bubbles will grow and then collapse. Based on this problem, the explicit Euler's method, implicit Euler's method, modified Euler's method; runge - kutta method and ODE45 method are introduced and compared for solving the ODE problem. All these methods are consistent with each other in solving the $2^{\text {nd }}$ order ODE. The error of them is very small. Also PDE method is introduced for solving PDE equations. And finite element method (FEM) is implied in modeling the problem.

The paper is to strengthen the importance of mathematical methods for students in engineering courses. There are too many engineering problems waiting for being studied and solved.

\section{Acknowledgements}

The work is supported by: The Fundamental Research Funds for The Central Universities (2572014CB10)

\section{References}

[1] Rayleigh L. On the Pressure Developed in a Liquid during the Collapse of a Spherical Cavity[J]. Philosophical Magazine, 1917, 34: 94-98.

[2] E.Hairer, S.P.Norsett, G.Wanner. Solving Ordinary Differential Equations I - Nonstiff Problems[M]. Second Revised Edition. Springer press. 1992

[3] Sun Zhizhong. Numerical solutions for partial differential equations[M]. Science Publishing House, 2005.1

[4] David Bleecker, George Csordas. Basic Partial Differential Equations[M]. International Press of Boston, 1997

[5] Andrei D. Polyanin. HANDBOOK OF LINEAR PARTIAL DIFFERENTIAL EQUATIONS for ENGINEERS and SCIENTISTS (First Edition)[M]. CHAPMAN \& HALL/CRC. 2002 